\title{
A note on Steiner symmetrization of hyperbolic triangles
}

\author{
François Guéritaud
}

\begin{abstract}
François Guéritaud studiert Mathematik an der École Normale Supérieure in Paris. Bis Ende 2002 arbeitete er als Forschungsstudent an der University of Southern California in Los Angeles. Neben seinem Interesse an der Visualisierung von regelmässigen 4-dimensionalen Polytopen umfasst sein Arbeitsgebiet gegenwärtig die Topologie und die hyperbolische Geometrie.
\end{abstract}

We give a short proof of a statement first shown in [1, Thm 4] and also used in [2] for isoperimetric considerations about hyperbolic simplices. The original proof given in [1] involved some computer algebra. The statement is as follows:

Let us consider a geodesic line $D$ in the hyperbolic plane, together with two hypercycles $\Lambda$ and $H$ (curves at constant distance from $D$ ) lying in the same half plane, at distances $\lambda$ and $h$ from $D$ respectively, with $0<\lambda<h$. Then let $A, B \in D$ be fixed points and consider all triangles $A B C$ with $C \in H$. We claim that:

Theorem 1 The intersection of $A B C$ with $\Lambda$ is of maximal length when $A B C$ is isosceles with respect to $C$, and only then.

Bezeichnet man Halbkreise der oberen Halbebene der komplexen Zahlenebene, die senkrecht auf der reellen Achse stehen, als „Geraden dieser Halbebene”, so erhält man ein Modell der hyperbolischen Geometrie (konstanter negativer Krümmung), das sogenannte Poincaré Modell. Die Längenmessung in dieser Geometrie unterscheidet sich deutlich von der uns vertrauten Längenbestimmung in der Euklidischen Geometrie. Dementsprechend komplex gestalten sich in der Regel Längen- und Flächenbestimmungen in der hyperbolischen Geometrie. Trotz dieses Umstands gelingt dem Autor in dieser Arbeit ein elementarer Beweis einer Maximalitätseigenschaft gleichschenkliger hyperbolischer Dreiecke, aus der sich leicht verschiedene isoperimetrische Ungleichungen ableiten lassen. In einem früheren Beweis desselben Satzes mussten L. Karp und N. Peyerimhoff noch auf eine Faktorisierung eines Polynoms vom Grade 38 mit Hilfe eines Computeralgebrasystems zurückgreifen. 


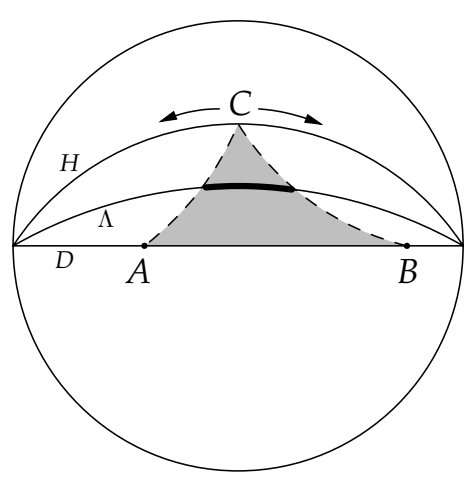

We shall prove this in the upper half plane model, taking $D$ to be the geodesic line through 0 and $\infty$ (hence $H$ and $\Lambda$ will appear as Euclidean half-lines, or rays, through $0)$. Let us first prove the following

Lemma Let $O \Omega$ be a line in the Euclidean plane and let $X, Y, Z$ lie on a circle centered at $\Omega$. Project $X, Y, Z$ onto $O \Omega$ and denote by $x, y, z$ the corresponding linear coordinates with respect to $O$. Then

$$
S:=(x-y) \overrightarrow{O Z}^{2}+(y-z) \overrightarrow{O X}^{2}+(z-x) \overrightarrow{O Y}^{2}=0
$$

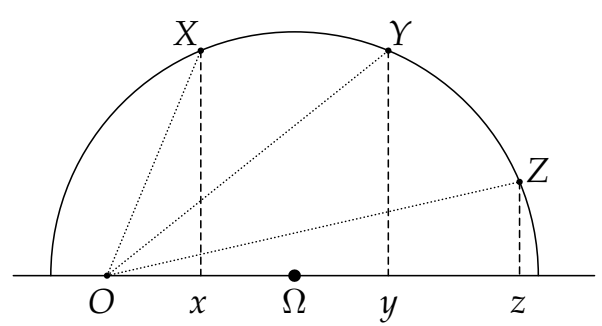

Indeed, we have

$$
\begin{aligned}
S & =(x-y)(\overrightarrow{O \Omega}+\overrightarrow{\Omega Z})^{2}+(y-z)(\overrightarrow{O \Omega}+\overrightarrow{\Omega X})^{2}+(z-x)(\overrightarrow{O \Omega}+\overrightarrow{\Omega Y})^{2} \\
& =0+2 \overrightarrow{O \Omega} \cdot((x-y) \overrightarrow{\Omega Z}+(y-z) \overrightarrow{\Omega X}+(z-x) \overrightarrow{\Omega Y})+0 \\
& =2 \omega((x-y)(z-\omega)+(y-z)(x-\omega)+(z-x)(y-\omega)) \\
& =0
\end{aligned}
$$

where $\omega$ denotes the linear coordinate of $\Omega$. Now in order to prove Theorem 1 , let us first replace it by the following, equivalent statement:

Theorem 2 Among all triangles with fixed apex $C \in H$ which intercept a given length $\ell$ of the hypercycle $\Lambda$, the one with shortest basis on $D$ is the isosceles one. 
The equivalence clearly follows from the monotonicity of the end abscissae of the arc intercepted in $\Lambda$, according to the end abscissae of the arc intercepted in $D$.

Let $(\cos \theta, \sin \theta)$ be the coordinates of the point $C$ in the upper half plane model, with $0<\theta<\pi / 2$ (up to an isometry fixing 0 and $\infty, C$ can indeed be taken on the unit circle). Now $H$ is the Euclidean ray through 0 and $C$, making an angle $\theta$ with the horizontal direction, and $\Lambda$ is another ray through 0 making an angle of, say, $\beta$ where $\theta<\beta<\pi / 2$. A point $M$ running on $\Lambda$ at constant speed has coordinates $\left(e^{t} \cos \beta, e^{t} \sin \beta\right)$ and, if the hyperbolic line through $C$ and $M$ intersects $D$ at Euclidean height $s$, we have by the lemma:

$$
s^{2}\left(\cos \theta-e^{t} \cos \beta\right)+e^{2 t}(0-\cos \theta)+1 \cdot\left(e^{t} \cos \beta-0\right)=0,
$$

i.e. (setting $0<p=\cos \beta<q=\cos \theta$ ):

$$
s^{2}=s^{2}(t)=\frac{e^{2 t} q-e^{t} p}{q-e^{t} p}=e^{t} \cdot \frac{e^{t} q-p}{q-e^{t} p} .
$$

Note that this quantity is positive, i.e., $e^{t} q^{2}+e^{t} p^{2}-p q-e^{2 t} p q>0$ or, equivalently:

$$
p^{2}+q^{2}>2 p q \cosh t
$$

Now the interesting quantity, on which the length of the basis depends (strictly) monotonically, appears to be (given some $\delta>0$ ):

$$
\begin{aligned}
\nu(t) & =\frac{s^{2}(t+\delta)}{s^{2}(t-\delta)} \\
& =e^{2 \delta} \cdot \frac{e^{t+\delta} q-p}{q-e^{t+\delta} p} \cdot \frac{q-e^{t-\delta} p}{e^{t-\delta} q-p} \\
& =e^{2 \delta} \cdot \frac{e^{t+\delta} q^{2}+e^{t-\delta} p^{2}-p q\left(e^{2 t}+1\right)}{e^{t-\delta} q^{2}+e^{t+\delta} p^{2}-p q\left(e^{2 t}+1\right)} \\
& =e^{2 \delta} \cdot \frac{e^{\delta} q^{2}+e^{-\delta} p^{2}-2 p q \cosh t}{e^{\delta} p^{2}+e^{-\delta} q^{2}-2 p q \cosh t} .
\end{aligned}
$$

Note that this last quantity has positive numerator (see (1) and the fact that $0<p<q$ ), and is positive: therefore the denominator is positive as well, and we have (omitting the factor $e^{2 \delta}$ ) the quotient of two positive quantities whose difference is constant, the numerator being bigger (recall again that $0<p<q$ ). Such a quotient is strictly decreasing with its numerator, and thus is minimal iff $t=0$. It should be added that if any value of $t$ at all does indeed satisfy $p^{2}+q^{2}>2 p q \cosh (t \pm \delta)$, i.e., $\nu(t)$ well-defined, then $t=0$ does. Since the hyperbolic line $C M$ appears in that case to be the unit circle, which is perpendicular to the geodesic line $0 \infty$, we have proved Theorem 1 . 


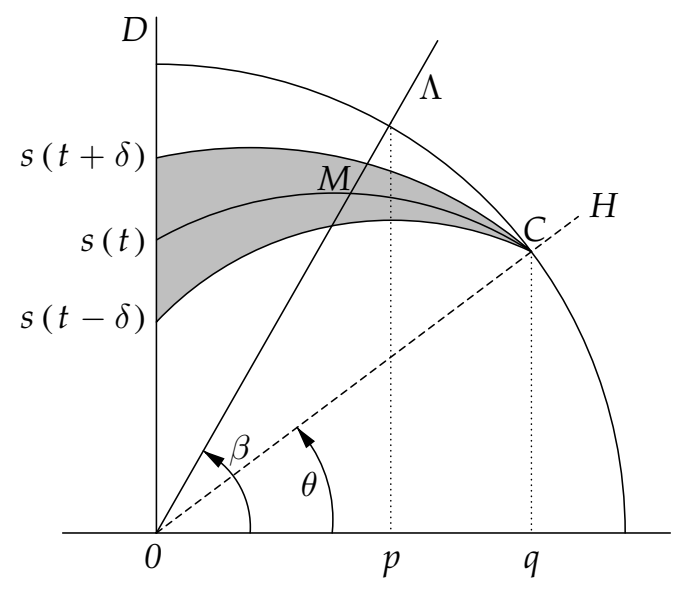

Note on Steiner symmetrization: Steiner symmetrization in dimension 2 is the areapreserving process by which a given object is cut into infinitesimal, parallel slices of constant width, each slice being then centered relative to an axis running orthogonally through the pile of slices. The slice separators in the hyperbolic framework, as introduced in [4], are parallel hypercycles, and in the Euclidean plane they are just parallel straight lines; in either case, the area-preserving property can be seen by applying Fubini's theorem.

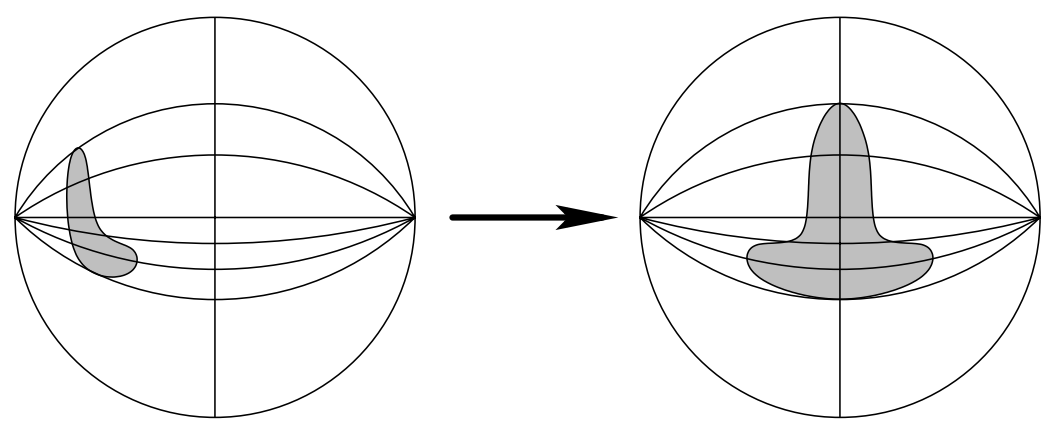

Fig. 1 Hyperbolic Steiner symmetrization

It is a well-known fact that Euclidean Steiner symmetrization preserves convexity. We have shown (thus answering a question stated in the introduction of [3]) that this does not hold any more in the hyperbolic plane, since a symmetrized hyperbolic triangle appears to be strictly contained in its convex hull (the hull is the isosceles, "optimal" triangle which simultaneously maximizes width at every intermediary height).

Acknowledgements. I wish to thank Norbert Peyerimhoff for his careful re-reading and many suggestions. 


\section{References}

[1] Karp, L.; Peyerimhoff, N.: Extremal properties of the principal Dirichlet eigenvalue for regular polygons in the hyperbolic plane, Archiv der Mathematik, Birkhäuser Verlag, 79 (2002).

[2] Peyerimhoff, N.: Simplices of maximal volume or minimal total edge length in hyperbolic space, $J$. London Math. Soc., Cambridge Univ. Press (2) 66 (2002).

[3] Schneider, J.: Über die Symmetrisierung kompakter Mengen im hyperbolischen Raum, Dissertation, Universität Stuttgart, 1986.

[4] Schneider, J.: Zum isodiametrischen Problem im hyperbolischen Raum, manuscripta mathematica 60 (1988)4, 437-461.

François Guéritaud

École Normale Supérieure

45 rue d'Ulm

F-75005 Paris, France

e-mail: gueritau@clipper.ens.fr 